\title{
El mundo como un taller de observación. La creación del Departamento Nacional del trabajo y las influencias internacionales ${ }^{1}$
}

por

Juan Suriano

Universidad Nacional de San Martín

\begin{abstract}
El presente trabajo analiza cuales fueron las experiencias internacionales que influyeron sobre la formulación de las primeras respuestas ensayadas por el Estado argentino en materia de legislación laboral. La ausencia de un corpus de conocimientos especializados (estadisticas fiables, informes, datos cualitativos) condujo a los sectores preocupados por resolver la cuestión obrera a estudiar y observar las prácticas y los métodos desarrollados en otros países. La experiencia adquirida en ese taller de observación mundial, en un contexto de fluida circulación internacional de las ideas, modelos de análisis y teorías, permitió pensar y poner en práctica las primeras leyes obreras así como crear el primer organismo laboral en el país.

Palabras Clave: Cuestión social; Legislación; Circulación de ideas; Conocimiento; Códigos; Políticas laborales.
\end{abstract}

Este artículo analiza cuáles fueron las experiencias internacionales que influyeron sobre la formulación de las primeras respuestas ensayadas por el Estado argentino en materia de legislación laboral. La ausencia de una tradición local en materia legislativa así como de la inexistencia de un corpus de

\footnotetext{
${ }^{1}$ Este trabajo es parte de dos proyectos: 1) PICT 02248, Historia de las instituciones laborales en Argentina: del Departamento Nacional del Trabajo al Ministerio del Trabajo, 1907-1955, financiado por la Agencia Nacional de Promoción Científica y Tecnológica, Argentina; 2) HAR, 2009-13555 Circulación internacional de saberes y prácticas institucionales en la consolidación del Estado Social en Argentina (1920-1970), financiado por el Plan Nacional I + D, Ministerio de Economía y Competitividad, España.
} 
conocimientos especializados (estadísticas fiables, informes, datos cualitativos) condujo naturalmente a los sectores preocupados por resolver la cuestión obrera a estudiar y observar las prácticas y los métodos desarrollados en otros países. La sólida experiencia que se fue adquiriendo en ese taller de observación internacional, ya fuera desde el seno del propio Estado o desde el estudio universitario, permitió pensar y poner en práctica las primeras leyes obreras, así como crear el primer organismo laboral en el país. Por otra parte este proceso se llevó adelante en un contexto de fluida circulación internacional de las ideas, modelos de análisis y teorías².

A partir de la crisis económica (y social) que afectó a Argentina desde 1890, comenzaron a hacerse evidentes los síntomas de diversos malestares y problemas existentes en el mundo del trabajo (desocupación, inestabilidad, problemas salariales, condiciones de trabajo, costo de vida). Este se había conformado recientemente como consecuencia de la integración del país al nuevo orden económico internacional, y la crisis desnudó serios desajustes que en la época se conoció como «cuestión obrera». La situación fue percibida rápidamente por los entonces escasos socialistas que también hacían su irrupción en ese momento. A través de su periódico, El Obrero, ponían en evidencia los graves problemas que vivían los trabajadores, como la caída de los salarios, el aumento de la explotación, la pérdida de empleo y, lógicamente, el retorno obligado de muchos de los inmigrantes ${ }^{3}$. Si bien la crisis fue coyuntural, la "cuestión obrera» fue adquiriendo mayores dimensiones en la medida en que la economía argentina crecía aceleradamente. El Estado demoró un tiempo más en descubrir la magnitud del problema y lo hizo cuando estallaron las grandes huelgas al despuntar el siglo XX. Pero a partir de ese momento la resolución de la «cuestión obrera» comenzó a ocupar un lugar importante dentro de las preocupaciones de algunos hombres de Estado, así como parlamentarios, intelectuales y profesionales ${ }^{4}$.

La búsqueda de soluciones a los problemas suscitados en el mundo del trabajo encontró una serie de escollos importantes entre los cuales se destacaba claramente la ausencia de un campo conocimientos al respecto. Al momento de crearse el Departamento Nacional del Trabajo (DNT) en 1907, no existía un corpus de investigaciones sobre las condiciones de trabajo, más allá de algunas notas periodísticas que, generalmente en tono de denuncia, solo brindaban datos fragmentarios. Dentro de la producción periodística se destaca claramente una serie de cuarenta artículos titulada «Los obreros y el trabajo», publicada

\footnotetext{
${ }^{2}$ Al respecto, véase Glick, 1987.

3 González Leandri, González Bernaldo y Suriano, 2010: 156-163.

${ }^{4}$ Suriano, 2000.
} 
por el diario La Prensa entre agosto y octubre de 1901. Este matutino venía alertando hacía tiempo sobre diversos aspectos negativos de la vida obrera; lo había hecho sobre las pésimas condiciones de la vivienda desde la década de 1870 y la situación obrera desde la crisis de 1890. En la serie de 1901 se abordan muchos de los temas (costo de vida, condiciones de trabajo y de vida, salarios, etc) que años más tarde serían materia de atención del $\mathrm{DNT}^{5}$.

A diferencia de lo que ocurría en algunos países como Alemania, Estados Unidos, Francia o Inglaterra en donde la adopción de políticas sociales por parte del Estado podía apoyarse en largas series de investigaciones parlamentarias o universitarias previas ${ }^{6}$, en Argentina, al momento de crearse el Departamento Nacional del Trabajo (DNT) en 1907, el campo de producción en este tema era de escasa densidad. Los datos que podían aportar los censos nacionales (1869 y 1895) y los de la ciudad de Buenos Aires (1888 y 1904) solo brindaban una base estadística (no siempre confiable) para conocer la cantidad de trabajadores y su distribución por sector y región. Las fuentes disponibles provenían de, al menos, tres espacios diferentes.

En primer lugar, debe destacarse que fueron los socialistas los primeros en llamar la atención sobre los problemas existentes en el mundo del trabajo, desde las tempranas advertencias a fines de la década de 1880 del periódico Vorwärts, escrito en idioma alemán, hasta las denuncias efectuadas a comienzos de la década siguiente por El Obrero o la información aparecida en $L a$ Vanguardia desde 1894. Fueron precisamente los socialistas, a través de un Comité Internacional Obrero, quienes, reflejando las decisiones de la Segunda Internacional, plantearon por primera vez al Parlamento la solicitud de «leyes protectoras de la clase obrera» ${ }^{7}$. Con la creación del Partido Socialista en 1896, se intensificaron los estudios relacionados con los problemas del mundo del trabajo, y un año después se publicó la investigación autodidacta de Adrián Patroni sobre las condiciones salariales de los trabajadores argentinos ${ }^{8}$. La

5 González, 1984.

${ }^{6}$ Es interesante observar el caso de Estados Unidos. Cuando en 1884 se creó el Bureau of Labour de carácter nacional lo precedían 15 departamentos laborales creados en otros tantos estados norteamericanos desde 1869. La masa de publicaciones de esas instituciones con informaciones y estadísticas del mundo del trabajo era impresionante, al punto de que Ernesto Quesada las calculaba en «al menos medio millar de volúmenes». Véase Quesada, I/119, (Buenos Aires, 1907).

${ }^{7}$ El Obrero, 7 de febrero de 1891. Es importante recalcar que algunos de los temas puntuales de dichas demandas se anticiparon en más de 10 años a la agenda de temas incluidos en la políticas laborales del Estado (limitación de la jornada de trabajo, regulación del trabajo femenino e infantil, inspección de de talleres y fábricas, abolición del trabajo nocturno, seguro obligatorio contra accidentes de trabajo, etc.).

8 Patroni, 1897. 
llegada de Alfredo Palacios, el primer diputado de ese partido, al Congreso Nacional en 1904, significó un salto cualitativo importante. Abogado recién recibido, fue un gran impulsor de la sanción de una legislación protectora de los trabajadores. En sus intervenciones en el Congreso combinaba el tono de denuncia típico de las alocuciones parlamentarias con fundamentos de una mayor rigurosidad propia de los ámbitos académicos. También debe destacarse el aporte de José Ingenieros, quien planteaba que la resolución de la cuestión social no pasaba ni la represión ni la acción de la caridad tradicional. Según su criterio, debía ser el Estado el encargado de educar a los trabajadores y resolver los problemas a través de una legislación adecuada, y no en vano participaría activamente de la redacción del proyecto de ley nacional del trabajo. En 1906 daría a conocer su libro sobre la legislación laboral ${ }^{9}$.

En el mismo sentido de los aportes documentales del socialismo, deben considerarse los reclamos de las federaciones gremiales y los periódicos que los representaban, como Anales de la Sociedad Tipográfica Bonaerense, El Obrero Panadero, La Unión Gremial, El Carpintero, El Mecánico o El Pintor, todos aparecidos antes de 1900. Estos periódicos sistemáticamente denunciaban las excesivas jornadas de trabajo, la desocupación, las condiciones del trabajo femenino e infantil o la falta de seguridad en los lugares del trabajo, pero también interpelaban y reclamaban al Estado que encarara la legislación laboral ${ }^{10}$.

El segundo espacio de producción de investigación y conocimiento, menos informal que el anterior, lo constituía el ámbito universitario, particularmente la Facultad de Derecho, y la - aún incipiente - carrera de sociología. En el primer caso, hubo una preocupación temprana por la cuestión social en vinculación con el derecho criminal, debido a la influencia de la criminología positivista. Por otro lado, los problemas sociales se abordaban desde los límites del derecho civil para resolver cuestiones como la responsabilidad de los empleadores en los accidentes de trabajo ${ }^{11}$. A partir 1898 comenzaron a producirse tesis doctorales vinculadas a aspectos jurídicos de la cuestión social (naturaleza jurídica del contrato de trabajo, responsabilidad legal de los accidentes laborales, análisis jurídico de la legislación laboral y social, arbitraje y conciliación en los conflictos entre el capital y el trabajo) ${ }^{12}$. Con respecto a la

\footnotetext{
9 Ingenieros, 1906. Sobre las ideas de Ingenieros en este tema, véase Terán, 1987.

${ }^{10}$ Lobato, 2009.

11 Zimmermann, 1995: 91-95.

12 Cuando se creó el DNT se habían publicado las tesis de Federico Figueroa (Las huelgas en la República Argentina y el modo de combatirlas, Imprenta Tragant, Buenos Aires, 1906), Alejandro Russo (La legislación obrera, Buenos Aires, 1906), Condomí Alcorta (Legislación del trabajo), Salvatierra (Accidentes de trabajo), Singulany (Las huelgas ante el derecho
} 
sociología, su aparición como disciplina de la mano, entre otros, de Ernesto Quesada, quien inauguró la primera cátedra de esa materia en 1904, está vinculada a la búsqueda de criterios científicos destinados a pensar la resolución de la cuestión social a través de un programa de reformas ${ }^{13}$. Mucho más que la sociología, el derecho laboral realizaría en el futuro aportes fundamentales al proceso de construcción del Estado social, dotándolo de expertos y funcionarios así como de las bases jurídicas de la justicia laboral ${ }^{14}$.

El tercer ámbito de recopilación y producción de información sobre las condiciones de trabajo era el Parlamento. Me refiero específicamente al ámbito de la Cámara de Diputados en los momentos de presentación de proyectos de ley que, por supuesto, se enriquecían durante los debates. Los proyectos más importantes que aportaron importantes insumos a los futuros funcionarios laborales estatales fueron los de descanso dominical y de regulación del trabajo femenino e infantil aprobados respectivamente en 1905 y 1907; en el mismo nivel de importancia se puede mencionar los largos debates en torno al proyecto de ley de accidentes de trabajo que se aprobó en 1915, pero que tuvo su primer intento en 1902 cuando los diputados Belisario Roldán y Marco Avellaneda presentaron el primer proyecto, al que siguieron otros presentados por los diputados Alfredo Palacios (1906), A. Escobar (1910 y 1912), Rogelio Araya (1912 y 1914), Arturo Bas (1914) y nuevamente Palacios (1915).

Con ser importante, toda esta producción ${ }^{15}$ no alcanzaba ni remotamente a satisfacer las demandas necesarias tanto para la elaboración de un Código de Trabajo, como el que pretendía llevar adelante en 1904 el entonces ministro del interior Joaquín V. González, como tampoco sería suficiente para los primeros diagnósticos que debía efectuar el DNT a partir de 1907. La información existente era poco precisa, fragmentaria, incompleta y carecía de sistematicidad. Por otra parte, lo poco que se conocía correspondía casi de manera abrumadora a la ciudad de Buenos Aires, y se ignoraba el estado de las condiciones del mundo del trabajo en las provincias y territorios nacionales.

Podría decirse que, tomando los tres ámbitos en su conjunto, no existía aún un campo de producción de conocimiento sistemático y sólido sobre la cuestión social. En este punto me parece pertinente recurrir a una idea de Carlos Altamirano que, aunque referida a la sociología, puede extenderse

constitucional y su legislación), Marcenaro (La organización profesional). Véase Quesada, I/115, (Buenos Aires, 1907).

13 Zimmermann, 1995: 83-91.

${ }^{14}$ Importante en el mismo sentido fueron los aportes de la medicina social que se nuclearían en torno del Departamento de Higiene. Véase González Leandri, 1999 y 2010; Recalde, 1997.

${ }^{15}$ A la que se puede agregar trabajos como los realizados por Juan Alsina, especialista en temas de inmigración, sobre las condiciones de vida de los trabajadores. Alsina, 1905. 
a todo el campo de producción sobre lo social: «En Argentina, como en el conjunto de América Latina, los modos de descripción y de interpretación del mundo social que llamamos sociológicos no fueron producto de un reflexión endógena, y no podría hablarse del surgimiento, sino más bien del ingreso, la adopción y, eventualmente, la adaptación de esas formas todavía nuevas del discurso sobre la vida social» ${ }^{16}$.

Para quienes pretendían hallar una solución racional a la «cuestión obrera», se tornaba absolutamente necesario estudiar, observar y aprender de aquellas experiencias que se estaban llevando a cabo desde hacía más o menos tiempo en otros países, fueran estos naciones «nuevas» como Australia, Canadá, Nueva Zelandia o Estados Unidos, o los viejos estados con una tradición más antigua como Inglaterra, Francia, Bélgica, España, Italia o las naciones escandinavas. El mundo se había convertido para estos pioneros del estudio social en un verdadero taller de observación, era allí en donde había que abrevar comparando las diversas experiencias con el objeto de construir el laboratorio social del cual debía nacer un corpus de legislación asocial. El conocimiento del panorama social internacional que fueron adquiriendo los noveles funcionarios los iba convirtiendo en expertos y, en ocasiones, llegaron a alcanzar un excepcional grado de erudición. No solo conocían las políticas laborales de los estados centralizados, sino también de aquellas naciones con un alto grado de federalismo, y sabían de las políticas aplicadas en los distintos estados norteamericanos así como de la heterogeneidad de dichas políticas en los cantones suizos.

El saber en el taller internacional se adquiría a través de diversas estrategias; una de ellas era el contacto con instituciones académicas extranjeras así como las visitas exploratorias a las instituciones laborales de aquellos países que poseían una tradición en materia de legislación social. Un buen ejemplo al respecto es el diálogo intelectual que se estableció entre Joaquín V. González (primero, como funcionario del gobierno, y desde 1906 como presidente de la Universidad de La Plata) y el Grupo de Oviedo perteneciente a la universidad homónima. Dos de sus miembros, los abogados Adolfo Posada (que visitó Buenos Aires en 1910 invitado por el propio González) y Adolfo Alvarez Buylla, fueron pioneros en el estudio de la cuestión social peninsular, y tras fracasar en la creación de un Instituto del Trabajo en 1902 desempeñarían, a partir de 1904, un rol importante en el Instituto de Reformas Sociales de España. El contacto entre las partes fue por demás fluido y tanto Posada como Buylla prestaron especial atención al proyecto de ley nacional del trabajo elaborado por González y lo analizaron en las publicaciones del Instituto ${ }^{17}$.

\footnotetext{
16 Altamirano: 2004, 31.

17 Prado, 2010: 36-37; Zimmermann, 1995: 73-74.
} 
Reducido a quienes podían hacerlo, por fortuna personal o en misión para el gobierno, los viajes al exterior para visitar diversas instituciones o bibliotecas especializadas eran otro camino para la adquisición de saberes especializados. Un caso paradigmático es el de Marco Avellaneda quien, antes de asumir la presidencia del DNT en 1909, realizó una larga visita a distintas instituciones de Francia y España. En París se entrevistó con F. M. Viviani, ministro de trabajo francés, quien lo puso al tanto de la legislación laboral vigente, el funcionamiento del ministerio y los planes de construcción de viviendas baratas encarados por la Fundación Rothschild. En Madrid desarrolló una intensa visita al ya mencionado Instituto de Reformas Sociales, con el que estaba familiarizado por la lectura del libro que Adolfo Buylla, Adolfo Posada y Luis Morote habían escrito sobre el Instituto ${ }^{18}$. Avellaneda se entusiasmó con el funcionamiento de las bibliotecas del Museo Social de París y del Instituto Reformas Sociales de Madrid, y sugirió la posibilidad de que el DNT creara una biblioteca similar con el objeto de atraer a los trabajadores. "Ayer nomás -decía- me decía el maestro Posada que nada había atraído y vinculado más al obrero que esa biblioteca» ${ }^{19}$.

Pero sin duda la principal forma de acceder al conocimiento de la producción del «taller internacional» era a través de la lectura de bibliografía especializada sobre la legislación obrera extranjera, fuera esta producción académica o de instituciones laborales. Por lo general se trataba de una bibliografía actualizada, leída generalmente en su idioma original o, a veces, en versiones traducidas al castellano. El mejor ejemplo - y por ello le dedico especial atención - para comprender la circulación de estos textos en el ámbito local y las formas en que fueron leídos e interpretados, lo encontramos en los fundamentos que anteceden y justifican el proyecto de Ley Nacional de Trabajo preparado por el Ministro del Interior, Joaquín V. González, y presentado al Parlamento el 6 de mayo de 1904. Para su elaboración, convocó a destacados intelectuales y profesionales - en su mayoría doctores en medicina y en jurisprudencia- entre los que se encontraban Armando Claros, el doctor Carlos Malbrán del Departamento Nacional de Higiene, el catalán Juan Bialet Massé, quien realizó un minucioso informe sobre el estado de «las clases obreras» en el interior del país ${ }^{20}$, así como los socialistas Augusto Bunge, Leopoldo Lugones, Manuel Ugarte, José Ingenieros, Enrique del Valle Iberlucea y el joven abogado Pablo Storni, quien se encargo de investigar y

\footnotetext{
18 Buylla, Posada y Morote, 1902.

19 Avellaneda, 1919: 177.

20 Bialet Masse, 1904.
} 
redactar el informe sobre la situación de los trabajadores porteños ${ }^{21}$. Si bien la autoría formal del proyecto corresponde a González, quien lo firmaba y quien tuvo la idea de la iniciativa, la promovió y autorizó, puede considerársela también como una obra colectiva.

El proyecto, cuyo objeto apuntaba a resolver la cuestión obrera en todas sus dimensiones, era un verdadero compendio general de lo que debería ser la legislación laboral a juicio de González. Inspirado en diversos aspectos de la legislación extranjera, se dividió en 14 títulos que comprendían 466 artículos, y abarcaban temas como las condiciones requeridas a los extranjeros para ejercer una «industria, oficio o profesión», el contrato de trabajo y los intermediarios, accidentes de trabajo, duración de la jornada laboral, el trabajo a domicilio, el «de los indios», el de menores y mujeres, el aprendizaje, las condiciones de higiene y seguridad, asociaciones obreras y patronales, tribunales de conciliación y arbitraje, así como contemplaba también la creación de una oficina laboral ${ }^{22}$. En la presentación de los fundamentos del proyecto, González sostenía que el propósito del mismo era eliminar las agitaciones cada vez más crecientes del movimiento obrero, cuyo aumento y organización fueron paralelos al crecimiento de la industria, el comercio y la economía en general. Esta cuestión no era por supuesto privativa de la República Argentina, «cada ley dictada en Europa, en América del Norte y en las colonias australianas, ha sido una consecuencia de una honda convulsión revolucionaria, y de sangrientas sacudidas que han permitido la paz de los pueblos y obligado a los partidos conservadores a ceder y ampliar el campo de expansión de los derechos y de la vida de las clases trabajadoras ${ }^{23}$. La propuesta de sancionar una ley, al estilo de un código, que englobara todas las cuestiones del mundo del trabajo, evitaba tener que legislar de urgencia después de cada agitación obrera. A juicio de su autor, esta forma de legislar la cuestión obrera podría prevenir y evitar los graves movimientos huelguísticos como los que se habían producido en el país en 1901 y 1902.

Si bien en otros países no existían códigos al estilo del que se proponía aquí, se apelaba claramente a estudiar las diversas experiencias extranjeras y adaptarlas a las necesidades del país: «En cuanto a sus formas si alguna novedad puede contener el proyecto será la que resulte de la más acertada asimilación de ciertos preceptos ya establecidos en otras leyes a las condiciones propias de nuestro país o de nuestra industria, y acaso el de

21 Zimmermann: 178.

${ }^{22}$ Proyecto de Ley Nacional del Trabajo, Congreso Nacional, Buenos Aires, 1904: 108171.

${ }^{23}$ Ibidem: 75. 
constituir el primer ensayo de una codificación general y amplia de todas las leyes parciales e incompletas dictadas hasta ahora en otras naciones, si bien limitada por los caracteres propios de nuestra sociabilidad y de nuestra legislación en otros órdenes, en particular los de los códigos comunes vigentes $\gg{ }^{24}$. Debían, entonces, estudiarse aquellas experiencias con legislaciones laborales, como el caso francés, y también las de aquellas naciones con estructuras institucionales más federativas, como Australia, Suiza, Alemania o Estados Unidos. Pero en cuanto a la idea de un código que englobara toda la legislación obrera, la principal fuente de inspiración de González fue el proyecto de programa social presentado por el político liberal inglés Joseph Chamberlain en 1892, cuyos puntos centrales (regulación de la jornada laboral, tribunales arbitrales, accidentes de trabajo, pensiones por ancianidad, restricción a los inmigrantes indigentes) «bosquejan ya, todos los elementos de lo que el proyecto del poder ejecutivo llama ley nacional de trabajo, porque abarca todas las energías de la actividad nacional en la producción de riqueza colectiva» ${ }^{25}$.

$\mathrm{Al}$ recorrer los fundamentos del proyecto comprobamos que el conocimiento y la estrategia narrativa de los fundamentos utilizada por los redactores se apoya, al margen de las citas sobre numerosas leyes extranjeras ${ }^{26}$, en la lectura de una treintena de obras especializadas publicadas en Inglaterra, Estados Unidos, Bélgica, Australia, Francia y España, casi todas en idioma original; las únicas traducciones detectadas se refieren al libro de Carrol D. Wright, uno de los pioneros de las estadísticas laborales norteamericanas, que fue leído en su versión francesa ${ }^{27}$, y un artículo de Carlos Lessona traducido por Enrique del Valle Iberlucea ${ }^{28}$. Con respecto a la propuesta de Chamberlain y su relación con las demandas sindicales en Inglaterra, el proyecto se apoyó en la lectura de los textos de S. H. Hayes (Mr Chamberlain, his life and public career, editado en Inglaterra en 1903) y de G. Howell (Trade Unions, new and old in social question today, Londres, 1891).

24 Ibidem: 77.

25 Ibidem: 84.

26 Por ejemplo, sobre las leyes de accidentes de trabajo y seguros obreros se aludían las leyes alemanas, «quizá las más perfectas que existen», de 1887, 1889, 1891, 1896, 1900 y 1903, así como también la ley italiana de 1898 y la española de 1900. Ibidem: 85.

27 Se trata del libro The industrial Evolution of the United States publicado en 1887. Wright dirigió el departamento de estadísticas laborales de Massachusetts (1873-1878). Se convirtió en una autoridad en el tema y su influencia se hizo sentir también en el desarrollo de las estadísticas laborales en Inglaterra. Véase Leiby, 1960.

28 Lessona, Carlos, «El contrato de trabajo», Revista Jurídica y de Ciencias Sociales, XXI/1-2, t. 1 (enero-febrero de 1904). 
Es interesante constatar que el primer problema planteado en el código no se refiere a cuestiones de seguridad laboral, sino a aspectos vinculados al mantenimiento del orden social. En este sentido, el tema en debate se relacionaba centralmente a los límites que debían imponerse a la inmigración. El proyecto ratificará los fundamentos de la ley de Residencia sancionada en 1902 basados en los postulados de la criminología positivista que sostenía la necesidad de preservar la paz social controlando de alguna manera el flujo inmigratorio en sus facetas más negativas. Toda sociedad «debe tender a depurar sus propios elementos étnicos por un régimen de orden moral e higiénico regular, y por un sistema de selección de todo elemento extraño que se incorpora de afuera», por eso las leyes nacionales deben «realizar esta tarea de depuración y selección» de los inmigrantes que llegan a la Argentina. Esa depuración se refería a individuos indigentes, enfermos incurables, proxenetas, pero, especialmente, a los principales enemigos del orden jurídico, esto es, a los activistas gremiales anarquistas que en los conflictos recientes habían demostrado una gran capacidad de movilización y convocatoria entre los trabajadores, pero además cuestionaban e impugnaban de raíz todo andamiaje del sistema político institucional de la nación. Estos hechos no habían sido previstos cuando se sancionó la Constitución Nacional hacía ya medio siglo y, aunque la ley de inmigración de 1876 preveía una limitación basada en principios de moral y salud pública, en la práctica los resultados fueron poco sustanciales. Si bien los principios liberales que guiaron el impulso a la inmigración no estaban en discusión, debía reformularse el principio de libertad absoluta imperante hasta aquí ${ }^{29}$.

El proyecto buscó la resolución de esta cuestión estudiando algunas experiencias «de otras naciones tan jóvenes como Argentina» con problemas parecidos, puesto que eran países de inmigración. Se enfocó especialmente el ejemplo de Estados Unidos, pues había sostenido un sistema inmigratorio tan permisivo como el argentino, aunque ahora sus dirigentes comenzaban a comprender los daños provocados por la libertad absoluta imperante en el ingreso de los inmigrantes. El principal inspirador intelectual de nuestros autores era el creador del sistema legal obrero de Nueva Zelandia, William Pember Reeves, a través de su libro State experiments in Australia and New Zeland (Londres, Grant and Richards, 1902), quien había sido un duro crítico de la excesiva liberalidad norteamericana, "actitud de una genial y abierta hospitalidad» pero sumamente negativa. Reeves había impulsado tempranamente en Australia y Nueva Zelandia «una legislación depuradora de su población adventicia». Así, el proyecto local estipulaba una serie de medidas preventivas

29 Proyecto de Ley Nacional del Trabajo, Congreso Nacional, Buenos Aires, 1904: 78-80. 
inspiradas en las «más perfectas leyes de los Estados Unidos (ley federal de restricción de 1903) y de las colonias inglesas de Oceanía», con el objeto de establecer el derecho por parte del Estado de no admitir a aquellos inmigrantes considerados indeseables por diferentes motivos ${ }^{30}$.

En el título dedicado a los accidentes de trabajo y el seguro obrero se reconocía que tanto el Código Civil como el Comercial ya no se adaptaban bien a las necesidades de protección de los trabajadores. Más allá de la admiración por las leyes alemanas, se reconocían otras influencias como el estudio crítico de la ley española de accidentes de trabajo, escrito por Hipólito González Rebollar, Adolfo Buylla y González Alegre publicado en Madrid en 1903, o el ya mencionado libro de Buylla, Posada y Morote sobre el Instituto del Trabajo español, con quien se estableció «un movimiento de honda simpatía» debido a «la campaña emprendida por el grupo intelectual de Oviedo, al amparo del ministro liberal, señor Canalejas, quienes se propusieron fundar el Instituto del Trabajo, como un núcleo inicial de grandes y trascendentales reformas a favor de la paz entre el capital y el trabajo». También se tuvieron en cuenta las distintas formas de seguro obrero adoptadas en Inglaterra expuestas en el libro de Charles Crawley (The law of insurance, S/F), así como los estudios realizados en Alemania, Italia, Francia y Bélgica ${ }^{31}$.

El proyecto prestó especial atención al contrato de trabajo, considerado por sus autores como la clave de la paz y armonía que debía existir entre trabajadores y patrones. Debía disponer las reglas para las prestaciones laborales (individuales o colectivas), la fijación del salario y la forma de pago y la relación entre las partes. La ley debía impedir las injusticias patronales y el consecuente «odio de clase» de sus empleados. El proyecto preveía que los mejores intermediarios para viabilizar los contratos de trabajo eran las agencias de colocaciones gratuitas del Estado, y seguían claramente el ejemplo de Francia, «donde se han hecho clásicas», Australia y Estados Unidos, a través de la información suministrada por los libros Le socialisme sans doctrines, de Albert Metin, y State activities in relation to labor in the U.S., de William Franklin Willoughby, ambos publicados en $1901^{32}$.

Con respecto a la duración de la jornada de trabajo se aconsejaba, como ya se había adoptado «por muchos estados de Europa y América», la jornada de ocho horas aunque, tomando como ejemplo las disparidades federales de Estados Unidos, se dejaba abierta la posibilidad de que cada provincia legislara

\footnotetext{
${ }^{30}$ Idem.

31 Ibidem: 85-87. Sobre las relaciones entre el reformismo liberal argentino y el grupo de Oviedo, véase Prado, 2006: 79-118.

${ }^{32}$ Ibidem; 87-90.
} 
el tema de acuerdo a sus propias características laborales. No obstante los redactores se muestran favorables a la implementación universal de la jornada de ocho horas y se apoyaban en el estudio de Juan Rac (La journée de huit heures, París, 1900) quien sostenía que «todas las reducciones de la jornada de trabajo, han hecho a la nación que las adoptó más sana, más rica y más sabia». Luego analiza los resultados de la aplicación de la jornada laboral en diversos gremios y estados de Europa, Estados Unidos y Australia. De manera lógica esta discusión estaba estrechamente vinculada al descanso dominical y a los días feriados no laborables y se realiza una detallada descripción de la legislación en Nueva Zelandia, Francia, Inglaterra y algunos cantones suizos. Como conclusión recomiendan el descanso dominical y adecuar los feriados a nuestra tradición religiosa y consuetudinaria como ocurría en otras latitudes ${ }^{33}$.

El trabajo a domicilio aparece en el proyecto como una preocupación mayor, dado que involucraba laboralmente al conjunto de la familia del trabajador y debido a las dificultades que entrañaba su control por parte del Estado. Amparado en el clásico principio liberal de la libertad del domicilio, se trataba de un ámbito de trabajo difícil de delimitar legalmente. Los reformadores buscaban una fórmula que compatibilizara los límites de la libertad de domicilio (el domicilio deja de ser inviolable cuando representa un peligro para la salud de la familia obrera y, por consecuencia, de la comunidad) y el derecho al trabajo inherente de cualquier individuo. Para articular las normas que debían reglar su actividad se estudiaron a fondo las legislaciones de Inglaterra, Estados Unidos y Australia, «los sistemas más ideales para evitar los inconvenientes del trabajo a domicilio», «sus fines son de indudable conveniencia: incorporar la pequeña industria al régimen de control higiénico de la industria general, y procurar acercar sus condiciones jurídicas del trabajo a las del contrato a jornal». El artículo sobre las condiciones de higiene en este tipo de labor se modeló sobre la base de la ley francesa de higiene industrial de 1893 y en relación a la cantidad máxima de miembros de una familia que podían trabajar en su casa. La inspiración provino de la ley del cantón suizo de Belle Ville de 1884 y de la ley inglesa de 1901. Una de las preocupaciones mayores de los autores del proyecto se relaciona con la inspección del «taller doméstico». Después de estudiar minuciosamente diversas legislaciones (la misma ley inglesa, la de Nueva Zelandia de 1901, la del estado de Massachusetts de 1891 y otros estados norteamericanos) concuerdan con el ya citado W. F. Willoughby que esta forma de trabajo debía separar en el interior de la vivienda los ámbitos de trabajo y de descanso y, fundamentalmente, estar sometido a una inspección y control permanente de la misma manera que el resto de trabajo industrial.

33 Ibidem: 90-94. 
Además de las fuentes citadas, nuestros codificadores también se apoyaron en los trabajos Adolfo Posada (Socialismo y reforma social, Madrid, 1904) y en la conferencia de Manuel Riviére «Vingt ans de vie sociales», incluida en el texto Idées sociales et faits sociaux (París, 1903) ${ }^{34}$.

La asociación obrera venía a representar otro problema complejo pero necesario de resolver, pues hasta ese momento no se había reglamentado su funcionamiento. En principio el proyecto reconocía que, al igual que el contrato de trabajo, el sindicato moderno no estaba contemplado en los «códigos comunes», aunque el derecho de asociación con «fines útiles» ya estaba reconocido en la Constitución Nacional; por eso la ley debía permitirlos, siempre y cuando no se opusieran a la carta magna y no coartaran las libertades ajenas ni subvirtieran el orden público, manteniendo inviolable la línea divisoria entre lo lícito y lo ilícito. Como las otras secciones, el proyecto «se ha inspirado en modelos celebrados en el mundo entero», tratando de tomar «todo lo mejor que aquellas mantienen con la idea de no impedir la legítima expansión del espíritu de unión entre los gremios». Contemplando las experiencias extranjeras, los efectos benéficos de la asociación gremial legalmente reconocida estaban a la vista: además de proteger todos los aspectos de la vida del trabajador, podían crear centros de estudio para el mejoramiento moral y espiritual de los trabajadores y, fundamentalmente, celebrar contratos colectivos de trabajo directamente con los empresarios eliminando la intermediación y la problemática figura del contratista. Las asociaciones obreras también debían cumplir un rol central en la adopción de los métodos de lucha, orientando a los obreros hacia el uso de medios pacíficos y graduales y evitando el uso de la violencia, que sería severamente castigada por la ley laboral ${ }^{35}$.

Ahora bien, ¿cómo evitar los conflictos violentos y las huelgas extremas? Pregunta fundamental, pues estas preocupaciones recorren todo el texto. Los autores del proyecto vuelven a recurrir a Carrol D. Wright, quien sostenía con convicción que la mejor y más eficaz herramienta en ese sentido era el arbitraje mediante tribunales constituidos por representantes patronales, obreros y del

34 Ibidem: 94-97.

35 Ibidem: 99-102. Las fuentes de inspiración sobre la regulación sindical y los métodos de lucha se basaron, entre otras, en las leyes de Francia (1901), Bélgica (1898), Nueva Gales del Sur (1901), Australia (1896) y Nueva Zelandia (1894, 1895, 1898). La bibliografía utilizada en este campo fue amplia: La legislation belge des unions profesionnelles de Raoul Briquet (París, 1900); Syndicats ouvriers, fédérations, bourses du travail de León de Seilhac (París, Armand Colin, 1902); Les conflits du travail de Ives Guyot (París, 1903); State experiment in Australia and New Zealand de W. P. Reeves (Londres, 1902); Industrial and Social Life de J. Stranchey (Londres, 1901); Industrial Democracy de Sidney y Beatrice Webb (Londres, 1897, T. I y II), y los textos ya citados de C. Wright y G. Howell. 
estado, cuyo objetivo no era impedir las huelgas sino reducir su número y gravedad. Citan textualmente a Wright porque, según nuestros autores, «define con precisión absoluta el pensamiento del proyecto en lo relativo al arbitraje industrial». El experto del departamento de trabajo de Massachusetts definía por «conciliación industrial» los intentos llevados adelante por las autoridades para allanar las dificultades surgidas en la relación del capital con el trabajo. Esta tarea de conciliación se fundaba en elevados principios de moral y religión. También se reconoce en el proyecto «que no hay originalidad alguna en el sistema elegido, si no es para simplificar procedimientos para adaptarlos a nuestros medios y necesidades y al estado en que las organizaciones obreras se hallan». Al igual que había ocurrido en los países más industrializados (Inglaterra, Francia, Australia, Alemania, Austria o Suiza), en Argentina la «sociedad y la industria han marchado más de prisa» que los cambios legales, y los conflictos entre el capital y el trabajo ya no podían resolverse con las estipulaciones jurídicas de «nuestros viejos códigos». La generalización del arbitraje así como del contrato de trabajo podrían evolucionar hacia reglas jurídicas hasta conformar un derecho del trabajo «que se abre camino amplio en la doctrina y en la legislación positiva» ${ }^{36}$.

Debe destacarse también la breve (un solo párrafo) pero importante referencia de la encíclica del Papa León XIII del 15 de mayo de 1891. En realidad, se trata de una muestra de beneplácito por el rumbo adoptado por la iglesia y un acuerdo con los principios sociales básicos allí estipulados: mejora en la situación personal de los trabajadores, tratamiento humanitario, mejor reparto de los frutos del trabajo a través de salarios dignos, derecho de asociación, instrumentación de la conciliación y la búsqueda de la paz entre el trabajo y el capital. Con mayor o menor precisión, los principios de la doctrina social de la iglesia se hallaban presentes de manera subyacente en los fundamentos del proyecto ideado por Joaquín V. González ${ }^{37}$.

La sanción del proyecto de ley impulsada por González se vio frustrada por dos motivos centrales: por un lado, la falta de interés de la mayoría de los legisladores de ambas cámaras del Congreso Nacional, que ni siquiera llegaron a tratarlo y debatirlo; por otro lado, se produjo una fuerte oposición de algunas entidades que agrupaban a los trabajadores orientados por el anarquismo (Federación Obrera de la Región Argentina) y a los empresarios (Unión Industrial Argentina). En el caso de la FORA se trataba de una clara muestra de oposición a cualquier injerencia estatal en la organización

${ }^{36}$ Ibidem: 105-108. Además de C. Wright, recurrieron al libro de A. J. Clark Studies in Australian Constitutional Law (Melbourne, 1901) y al artículo de Carlos Lessona (cf. nota 28).

${ }^{37}$ Ibidem: 85. 
obrera, pero de manera particular rechazaban tajantemente aquellos aspectos represivos vinculados a la limitación impuesta a la inmigración y a los condicionamientos referidos a la organización gremial, razones que llevaron al IV Congreso de la FORA a declarar que los contenidos del proyecto «son un atentado sin precedentes a todas las libertades colectivas e individuales $\gg{ }^{38}$. Por su parte, no dejan de ser interesantes los argumentos utilizados por los industriales para fundamentar su rechazo. «Muchas de sus transcripciones de leyes extranjeras - sostenían-, no son todavía aplicables en nuestro país», puesto que la industria presentaba aquí características diferentes a las de Europa. Si el proyecto se aprobara, «este ensayo lanzaría al país en un camino lleno de dificultades y de peligros cuyas consecuencias podrían ser de lo más funestas». Y el ejemplo utilizado también proviene de una experiencia exterior. En efecto, el gobierno debería tener en cuenta el fracaso de las leyes obreras sancionadas en Francia después de las convulsiones de 1848. Los industriales ponderaban las intenciones de los profesionales que redactaron el proyecto, «pero notoriamente adictas a las más avanzadas teorías y visiblemente poco conocedoras de nuestras modalidades de trabajo, de nuestro medio industrial». Objetaban, además, no haber sido consultados como había ocurrido con la legislación social en Alemania, Bélgica y Suiza, en donde se había pedido la opinión de todos los sectores interesados ${ }^{39}$.

No obstante las objeciones y como una derivación del proyecto, en los años siguientes se aprobaron las dos primeras leyes de protección obrera: en 1905, la número 4661 de descanso dominical, y dos años después, la 5291 de protección del trabajo femenino e infantil. Por otro lado, los debates sobre políticas laborales producidos de aquí en adelante abrevaron en numerosas ocasiones en el contenido del proyecto frustrado. Cuando en 1921 el presidente Hipólito Yrigoyen le encargó la redacción de un nuevo código laboral al Dr. Alejandro Unsain, funcionario del DNT desde 1901 y especialista en derecho del trabajo, este se basó en buena medida en los contenidos del proyecto González al que le limó los aspectos más represivos.

Nos interesa aquí el tercer subproducto surgido del malogrado proyecto. Este preveía en su Título XIII la creación de una Junta Nacional del Trabajo, bajo dependencia del Ministerio del Interior, que tendría a su cargo «el vigor

${ }^{38}$ Bilsky, 1985: T. 2, 206. Los gremialistas anarquistas Alberto Castro y Carlos García Balsas escribieron un panfleto titulado «Crítica al proyecto González», basado en la impugnación de la intervención estatal en las relaciones laborales. Suriano, 2000: 89-110.

${ }^{39}$ Congreso Nacional, Cámara de Diputados, Diario de Sesiones, Buenos Aires, 1905: 694-700. 
y dirección inmediata de las funciones que esta ley crea»..$^{40}$ En los fundamentos se recalca el retraso argentino en materia de políticas laborales al no disponer de una oficina de estas características en un momento en el que «no existe casi un solo estado civilizado donde no se haya establecido este servicio». El sentido de esta institución debía ser el estudio metódico de todas aquellas cuestiones vinculadas a las relaciones entre empresarios y trabajadores, así como las condiciones de vida y de trabajo de estos a través de la generación de estadísticas y de informes cualitativos que deberían publicarse sistemáticamente. Si bien tomaba como modelo el departamento de trabajo norteamericano creado en 1884, también prestaba atención a otros organismos como el Instituto de Reformas Sociales de España, que ponderaban para sí la capacidad de inspección y el poder de policía industrial ${ }^{41}$.

De allí se desprendió la propuesta de creación del Departamento Nacional del Trabajo, presentada en 1907 en el Congreso Nacional. Si sorpresiva fue la presentación también lo fue la rapidez con que fue aprobada, sin que se produjera un debate a fondo sobre las características que debía adquirir dicha oficina estatal. La presentación de la propuesta estuvo a cargo del diputado Julio Roca (hijo), quien puso como modelo al departamento de trabajo norteamericano. Las únicas observaciones fueron planteadas por el diputado socialista Alfredo Palacios, quien apoyó de manera entusiasta la iniciativa pero pidió más tiempo para debatir el sentido y las características que debía adquirir una institución laboral tan importante. En este sentido señaló que el modelo norteamericano no era el único a tener en cuenta: «ahí están la Inglaterra, que recientemente ha creado un ministerio, y la Francia revolucionaria y progresista, que marcha a pasos de gigante solucionando complejísimos problemas de orden social». Agrega a esta lista a Nueva Zelandia y Australia. Pero Palacios advierte a la Cámara que en dichos países la creación de oficinas laborales se hallaba precedida de una legislación social mediante la cual el estado velaba por el bienestar de la población, y la función de estos organismos era vigilar el cumplimiento de las leyes obreras. «Desgraciadamente nosotros estamos muy atrasados. Quizá hubiera sido conveniente que, antes que crear una oficina como la que se propone, se dictaran algunas leyes para que este departamento de trabajo tuviera qué hacer» ${ }^{42}$.

Finalmente se aprobó la propuesta y el doctor en jurisprudencia Nicolás Matienzo fue nombrado su primer presidente. El nuevo organismo estaba ins-

\footnotetext{
40 Proyecto de Ley Nacional del Trabajo, Congreso Nacional, Buenos Aires, 1904:162.

${ }^{41}$ Ibidem: 102-105.

42 Congreso Nacional, Cámara de Diputados, Diario de Sesiones, Buenos Aires, 1907: 638-646.
} 
pirado claramente en el pionero Departamento de Trabajo (Bureau of Labor) de Estados Unidos, organizado en Washington en 1884 y que al comienzo dependió, como ocurrió aquí con el DNT, del Ministerio del Interior; más tarde adquirió el rasgo de departamento independiente, aunque en 1903 perdió ese carácter para pasar a depender del Ministerio de Comercio. De una de sus publicaciones, el Bulletin of the bureau of labour, tomó el nombre el Boletín de nuestro $\mathrm{DNT}^{43}$. En el plan de trabajo elevado al ministro del interior, Manuel A. Montes de Oca, Matienzo reiteraba su admiración por el organismo norteamericano y señalaba con lucidez las carencias y los problemas que debía enfrentar la joven institución argentina. Reiteraba la ausencia de «materiales metódicamente acumulados», como existen en otros países (Estados Unidos, Inglaterra, Francia, Alemania, Bélgica, Australia, Nueva Zelandia) en donde las instituciones análogas funcionan desde muchos años atrás, recogiendo y coordinando informaciones relativas al trabajo y sus relaciones con el capital y con el estado, pero a la vez señalaba: «por valiosas que sean las indagaciones extranjeras, y por más que ellas recaigan sobre temas análogos a los que a nosotros nos preocupan, es decir, sobre formas y estipulaciones del contrato de trabajo y del de aprendizaje, sobre cuantía, límites y garantías del salario, sobre horas y épocas del trabajo, sobre descansos, sobre higiene, seguridad y moralidad en los talleres, sobre responsabilidad de accidentes de trabajo, sobre asociaciones y sindicatos, sobre seguros contra la enfermedad, la vejez y la invalidez, sobre arbitrajes entre obreros y patronos, sobre costo de producción de los artículos, sobre provecho obtenido por los fabricantes y productores, sobre el costo de vida, etc., etc.», por importantes que fueran esas indagaciones en el taller de observación internacional, «más indispensable es penetrar en el seno de nuestro propio pueblo y fijar con la precisión posible las modalidades que en él toman las múltiples relaciones de la vida en el trabajo» ${ }^{44}$.

Los primeros años de vida del DNT se vieron cruzados por un debate sobre las características y funciones que debía asumir la Oficina Laboral. Las posturas giraban en torno a dos modelos diferentes de organismo estatal que, a la vez, eran tributarios de otros tantos modelos internacionales: uno se inclinaba por la prescindencia de la institución y el otro era partidario de la intervención como policía industrial y comercial a través de la inspección de los establecimientos de trabajo para garantizar el cumplimiento de las leyes de laborales. El proyecto de Matienzo era partidario de la prescindencia y se inspiraba en la Oficina del Trabajo del gobierno federal norteamericano, que no reconocía esa competencia al organismo y se abocaba centralmente a la

\footnotetext{
${ }^{43}$ Quesada, I/ 1, 119-121, (Buenos Aires, 1907).

${ }^{44}$ Matienzo, I, 1, 23-24, (Buenos Aires, 1907).
} 
recopilación de estadísticas e informaciones (salarios, duración de la jornada laboral, condiciones de vida y de trabajo, precios de los productos de primera necesidad, etc.) y a la publicación de sus resultados. Desde un doctrinarismo liberal que rechazaba la intervención directa del Estado en las relaciones laborales (ámbito del derecho privado), Matienzo sostenía que «la policía industrial y comercial no forma parte de las atribuciones conferidas al DNT, porque no se aviene a la índole técnica e informativa de esta institución» y complicaría su función inherente; además perdería ella el carácter imparcial y sereno que es propio de todo estudio de reforma social, si se le agregase funciones de naturaleza persecutoria y represiva ${ }^{45}$. Sin embargo, su postura era minoritaria en relación a los funcionarios que lo acompañaban.

Quienes se mostraban favorables a la intervención activa del DNT tomaban diversos modelos como las oficinas del trabajo de Inglaterra (Labour Bureau) y Bélgica (Office du Travail). La primera permitía, según las Conciliation Acts de 1896, la mediación del organismo en los conflictos laborales y la imposición de multas a quienes infringían la decisión del organismo, mientras la segunda, creada en 1894, lo habilitaba en las funciones de inspección y vigilancia que, según el presidente del DNT, Julio Lezana, era una innovación belga ${ }^{46}$. También influyó en el mismo sentido el ya mencionado Instituto de Reformas Sociales creado en España en 1903 con quien los funcionarios argentinos de la institución seguían manteniendo una fluida relación. Cabe considerar una cuestión relevante en el caso argentino, pues la Ley Orgánica del DNT aprobada en 1912 disponía el rol arbitral del DNT, aunque de carácter voluntario, pues no imponía la obligatoriedad de la conciliación. Indudablemente, esta fue otra característica que limitaría la capacidad resolutiva del Departamento ${ }^{47}$.

A mediados de 1907, Ernesto Quesada dictó en la Universidad de La Plata la conferencia titulada «la cuestión obrera y su estudio universitario». Profesor de economía política en la Facultad de Ciencias Jurídicas y Sociales, Quesada era un firme propulsor de las Ciencias Sociales y estaba convencido de la importancia que en ellas tenía como materia de estudio la «cuestión obrera». El análisis de este tema en las aulas permitiría abordarlo con la mayor seriedad e imparcialidad. Uno de los temas centrales de su intervención se

45 Ibidem: 27.

46 Soprano, 2010: 91-92. También se prestó atención la Oficina de Trabajo francesa creada en 1891 y convertida en Dirección de Trabajo en 1900.

47 Años después, un importante funcionario del DNT, Alejandro Ruzo, volvió a insistir, amparándose en las experiencias de Bélgica, España y Francia, en la importancia de la inspección y el poder de policía del organismo. Véase Ruzo, 1918: 33-34; 138-146. 
refería a la trascendencia de la creación del DNT apenas unos meses antes ${ }^{48}$, y explicaba que la materia prima indispensable para su estudio era el material bibliográfico y documental reunido antes y después de la creación de las oficinas laborales en Estados Unidos (1884), Países Bajos (1889), Canadá (1890), Nueva Zelandia (1891), Alemania (1892), Inglaterra (1886), Francia (1891), Bélgica (1895), Austria (1898), Italia, Suecia y Noruega (1903) así como España (1904). Remarca las diferencias y similitudes en el funcionamiento de cada uno de ellos, pero llama la atención sobre una diferencia notable en la acumulación de «elementos de estudio anteriores» a la creación de dichas instituciones entre estas y el DNT argentino ${ }^{49}$. Tras un pormenorizado análisis del material bibliográfico, caso por caso existente en esos países, concluía que mientras en las naciones europeas la masa de publicaciones era «increíble», en Argentina, país más joven al fin, la producción era casi inexistente, pues no existía una tradición al respecto.

En todo caso, la única excepción como cuerpo de legislación social con valor documental había sido el proyecto de Ley Nacional de Trabajo presentado por el ministro González que, lamentaba, fue ignorado «deliberadamente» por el Parlamento pues, «salvo el único diputado socialista [...] nadie se mostró dispuesto a engolfarse en estudio semejante» ${ }^{50}$. Debido a este desinterés público por el problema obrero, Quesada decidió incorporar el tema en la cátedra de Economía Política de la Facultad de Ciencias Jurídicas y Sociales de la Universidad de La Plata, destinado a abogados recibidos. El insumo imprescindible de dicha materia estaría conformado por la producción bibliográfica de los trece departamentos laborales que existían en ese momento en el mundo. «De manera, entonces, que cualquier aspecto de la cuestión obrera, desde los contratos de salario, la duración de las jornadas, hasta las huelgas, paros y cierres, la situación moral y material obrera, sus sociedades, sus seguros, y cualquier modalidad de su legislación especial; todo, en una palabra, puede ser estudiado en sus aspectos típicos en casi todo el mundo civilizado, a la vez» ${ }^{51}$. La idea subyacente en el pensamiento de Quesada es que la producción bibliográfica internacional vinculada al estudio de los problemas locales contribuiría a generar un campo de producción para el conocimiento social de la cuestión obrera.

${ }^{48}$ No casualmente la conferencia fue reproducida en el primer número de la publicación del DNT, Quesada, 1907. Sobre la trayectoria de Quesada, véase Buchbinder, 2012.

49 Ibidem: 118.

${ }^{50}$ Ibidem: 112. Interesa destacar que Quesada fue muy crítico del informe de Bialet Masse, el más original de todos los que se habían presentado al ministro González, al que definió como «un esbozo lleno de defectos y vacíos».

${ }^{51}$ Ibidem: 144. 
En un contexto en donde la circulación de ideas a nivel internacional era muy fluida, los funcionarios del DNT estudiaban y conocían a fondo las políticas laborales en diferentes lugares del mundo. Los boletines publicaban infinidad de artículos sobre el tratamiento de temas como las formas de resolver los conflictos, las características de las instituciones laborales y todos los aspectos referidos a la legislación obrera existente en dichos países. Solo entre 1907 y 1912, se publicaron artículos sobre el tratamiento de dichos temas en Austria, Bélgica, Canadá, Inglaterra, Francia, Gales, Irlanda, Suecia, Estados Unidos, Alemania, España, Hungría, Finlandia, Suiza, Italia y Portugal. Esporádicamente, también aparecían algunos artículos sobre estas experiencias en algunos países sudamericanos. A partir del canje de publicaciones con las instituciones extranjeras, la llegada al país de expertos extranjeros, los viajes de sus funcionarios a congresos internacionales o la visita a instituciones laborales del exterior se establecieron redes transnacionales que enriquecieron de manera sustancial la formación de los funcionarios locales ${ }^{52}$. La adopción de conceptos y métodos de especialistas extranjeros, sumados a las observaciones y experiencias en el campo local, contribuyeron a conformar un dinámico campo de funcionarios especializados en conocimientos de cuestiones laborales.

Aunque excede los límites de este artículo, es interesante señalar que la activa participación argentina en estas redes aumentó los contactos internacionales y permitió un fructífero intercambio entre los expertos en el tema y las principales instituciones laborales del mundo. Este proceso dio sus frutos en 1919, cuando la recién constituida Sociedad de las Naciones $(\mathrm{SN})$ proyectó la creación de la Organización Internacional del Trabajo. En ese contexto, el presidente francés Georges Clemencau invitó a comienzos de 1920 formalmente a la Argentina integrarse a la SN y a participar de su creación en la Conferencia Internacional del Trabajo que se realizaría en Washington. El gobierno de Yrigoyen aceptó el convite y envió una delegación compuesta por un representante obrero y otro patronal, así como dos delegados del gobierno. Uno de ellos era el presidente interino del DNT, Alejandro Unsain, quien desempeñó una activa labor en dicha conferencia y sería incorporado como miembro del Consejo de Administración

${ }^{52}$ En ocasiones, como ya se ha recalcado, el DNT establecía relaciones más directas con instituciones extranjeras, como en el caso del Instituto de Reformas Sociales de Madrid durante la presidencia Marco Avellaneda, con cuyas orientaciones mantenía una fuerte afinidad al igual que con Adolfo Posada, uno de sus miembros prominentes. Esa influencia se hizo evidente en la redacción del frustrado proyecto de ley orgánica elaborado por Avellaneda (Zimmermann, 1995: 199. Bertolo, 2008: 72-73). No obstante, las huellas de esa influencia se plasmarían en la ley orgánica finalmente aprobada en 1912. 
de la OIT radicado en 1920 en Ginebra ${ }^{53}$. En realidad se estaba plasmando la concreción de un viejo anhelo de muchas organizaciones obreras y de algunas instituciones estatales de conformar normas comunes transnacionales de protección a los trabajadores, esto es, el derecho internacional obrero que se plasmaría en la OIT. Fue en este contexto e impulsados por dicha institución que se firmaron tratados de Argentina con España e Italia sobre reciprocidad en materia de indemnización por accidentes de trabajo, que se materializaron a través de las leyes $\mathrm{N}^{\mathrm{o}} 11.125$ y 11.126 sancionadas por el Congreso Nacional en $1920^{54}$.

Más allá de relativa importancia de estos acuerdos, este hecho señala los avances realizados en Argentina en materia de estudio y legislación laboral en el corto lapso de menos de dos décadas. En la base de este proceso los profesionales convocados por el ministro del interior en 1904 construyeron un verdadero taller de observación y estudio de la legislación extranjera. No puede pensarse este comienzo del proceso de construcción de las políticas laborales sin esa lectura de las experiencias internacionales que se profundizó en algunas cátedras universitarias. Era un proceso lógico pues la economía argentina formaba parte del mercado internacional y se regía por sus reglas; además, tanto las formas de organización del trabajo urbano, como los propios trabajadores (en gran número inmigrantes) y sus instituciones estaban modeladas en las experiencias previas desarrolladas en sus países de origen. Sin embargo, es importante señalar que no debe concluirse ligeramente que el proyecto González y la posterior acción del DNT se haya limitado a la mera copia de dichas experiencias, en principio porque todo proceso de recepción de ideas, teorías o modelos de interpretación es selectivo y no es pasivo. Tanto Marco Avellaneda como Ernesto Quesada, así como abogaban por dotar a las Ciencias Sociales de un carácter nacional, pensaban lo mismo con respecto a las políticas sociales ${ }^{55}$.

Solo que, como se ha sostenido al comienzo, era imposible pensar las políticas laborales locales sin tener en cuenta y referenciarse en las experiencias internacionales; la verdadera innovación de los codificadores y los funcionarios laborales consistió en efectuar las comparaciones de las diversas experiencias desarrolladas en otros países con el fin de encontrar aquello que a su juicio era

${ }^{53}$ Se dedicó un número completo del Boletín a reseñar la Conferencia de Washington. El informe fue redactado por Unsain. Véase $B D N T$, $\mathrm{N}^{\circ} 45$, febrero de 1920. Sobre la génesis del Derecho Internacional Obrero, véase Ruzo, 1918: 307-319. Palacios, 1920.

${ }^{54}$ En el artículo $1^{\circ}$ se establecía «que los ciudadanos de cada uno de los estados contratantes que fueran víctimas de accidentes de trabajo en otro estado, así como sus herederos, tendrán derecho a las indemnizaciones y demás excepciones que la ley nacional concede a los nacionales», en Alfredo Palacios, Derecho Internacional Obrero, Imprenta Mercatali, Buenos Aires, 1920: 32.

55 Avellaneda, 1919:14. Quesada, 1907: 113. 
lo más conveniente para construir la legislación laboral argentina ${ }^{56}$. Además, se prestó atención a las peculiaridades de la sociedad local, especialmente en las provincias del norte, como se estableció claramente en el informe de Bialet Massé, o también en las investigaciones e informes realizados entre 1913 y 1915 por el DNT sobre la explotación laboral en los quebrachales y yerbatales del interior del país, en donde las formas de organización del trabajo la resolución de ese problema adquirían características propias. En este sentido, también resultó significativo el estudio de los límites del derecho común observados en la Constitución de 1853, así como en los Códigos Civil y Comercial. De esta forma, partiendo de la observación y estudio en el taller internacional a la luz de las particularidades locales, se construyó el complejo laboratorio social argentino desde donde se fueron delineando las estrategias de las políticas laborales.

\section{BIBLIOGRAFÍA}

Alsina, Juan, El obrero en la República Argentina, Buenos Aires, 1905.

Altamirano, Carlos, «Entre el naturalismo y la psicología: el comienzo de la "ciencia social" en la Argentina», Federico Neiburg y Mariano Plotkin (eds.), Intelectuales y expertos. La constitución del conocimiento social en la Argentina, Buenos Aires, Paidós: 31-65.

Avellaneda, Marco, Del camino andado (economía social argentina), Buenos Aires, Cooperativa Editorial Limitada, 1919.

Bertolo, Maricel, Estado y trabajadores en Argentina. El Departamento Nacional del Trabajo ante el fenómeno de la desocupación, 1907-1934, Tesis de Doctorado, Facultad de Filosofía y Letras, Buenos Aires, 2008.

Bialet Massé, Juan, El estado de las clases obreras argentinas a comienzos del siglo, Buenos Aires, 1904.

Bilsky, Edgardo J., La Fora y el movimiento obrero, Buenos Aires, CEAL, 1985.

Buchbinder, Pablo, Los Quesada. Letras, ciencias y política en la Argentina, 18501934, Buenos Aires, Edhasa, 2012.

Buylla, Adolfo, Posada, Adolfo y Morote, Luis, El Instituto de Trabajo. Datos para la historia de la reforma social en España, Madrid, Establecimiento Ricardo Fe, 1902.

Glick, Thomas, «La transferencia de las revoluciones científicas a través de las fronteras culturales», Ciencia y Desarrollo, 12, (México,1987): 77-89.

\footnotetext{
${ }^{56}$ Alejandro Ruzo, quien fue uno de los propulsores de la creación de una justicia laboral autónoma, sostenía la importancia del estudio de la «legislación comparada» en países cuyos adelantos en este sentido eran notorios (caso especial, Francia) y establecer su relación con la prescripciones constitucionales y la codificación argentina. Ruzo, 1918: 38-41.
} 
González, Ricardo, Los obreros y el trabajo, Buenos Aires, 1901, Buenos Aires, CEAL, 1984.

González Leandri, Ricardo, González Bernaldo de Quirós, Pilar y Suriano, Juan, La temprana cuestión social. La ciudad de Buenos Aires durante la segunda mitad del siglo XIX, Madrid, CSIC, 2010.

González Leandri, Ricardo, Curar, persuadir, gobernar. La construcción histórica de la profesión médica en Buenos Aires, 1852-1886, Madrid, CSIC, 1999.

González Leandri, Ricardo, «Breve Historia del Departamento Nacional de Higiene, Estado, Gobernabilidad y Autonomía Médica en la segunda mitad del siglo XIX», un estado con rostro humano. Funcionarios e instituciones estatales en Argentina (desde 1880 hasta la actualidad), Ernesto Bohoslavsky y Germán Soprano (Eds.), Buenos Aires, Prometeo/Universidad de General Sarmiento, 2010: 59-85.

Ingenieros, José, La legislation du travail dans la Republique Argentine, París, Ed. Cornélly, 1906,

Leiby, James, Carrol Wright and Labor Reform. The origin Of Labor Statistics, Massachusetts, Harvard University Press, 1960.

Lobato, Mirta Zaida, La prensa obrera, Buenos Aires, Edhasa, 2009.

Matienzo, Nicolás, «Plan de trabajos», Boletín del Departamento Nacional del Trabajo, I/1 (Buenos Aires, 1907), 23-24.

Palacios, Alfredo, Derecho Internacional Obrero (El Congreso de Washington), Buenos Aires, Imprenta Mercatali, 1920.

Patroni, Adrián, Los trabajadores en la Argentina, Buenos Aires, 1897.

Prado, Gustavo, Las lecciones historiográficas de Rafael Altamira en Argentina (1909). Apuntes sobre Ciencia, Universidad y Pedagogía Patriótica, Oviedo, Universidad de Oviedo, 2010.

Prado, «Oviedo y La Plata, 1898-1910: el diálogo del reformismo liberal español y argentino en torno de la problemática hispano-americana», Pilar Cagiao Vila y Eduardo Rey Tristán (coords.), Aproximaciones al americanismo entre 1898 y 1936: Proyectos, Instituciones y Fondos de Investigación, Santiago de Compostela, Universidad de Santiago de Compostela, 2006.

Quesada, Ernesto, «La cuestión obrera y su estudio universitario», Boletín del Departamento Nacional del Trabajo, I/1 (Buenos Aires, 1907): 110-152.

Recalde, Héctor, La salud de los trabajadores en Buenos Aires (1870-1910). A través de las fuentes médicas, Buenos Aires, Grupo Editor Universitario, 1997.

Ruzo, Alejandro, Política Social, Buenos Aires, Talleres Gráficos L.J. Rosso, 1918.

Soprano, Germán, «El Departamento Nacional del Trabajo y su Proyecto de Regulación Estatal de las Relaciones Capital-Trabajo en Argentina, 1907-1943», José Panettieri (comp.) Argentina: trabajadores entre dos guerras, Eudeba, Buenos Aires, 2000. 
Soprano, Germán, «Del Estado en singular al Estado en plural. Contribución para una historia social de las agencias estatales en la Argentina», Cuestiones de sociología, Revista de Estudios Sociales, UNLP, La Plata, 2005.

Soprano, Germán, «Haciendo inspección. Un análisis del diseño y aplicación de la inspección laboral por los funcionarios del departamento Nacional del Trabajo (1907-1914)», Ernesto Bohoslavsky y Germán Sopran (eds.), Un Estado con rostro humano. Funcionarios e instituciones estatales en Argentina (desde 1880 a la actualidad), Prometeo Libros, Buenos Aires, 2010: 85-120.

Suriano, Juan, La cuestión social en Argentina 1870-1943, La Colmena, Buenos Aires, 2000.

Suriano, Juan, «El Departamento Nacional del Trabajo y la política laboral durante el primer gobierno de Hipólito Yrigoyen», Mariano Ben Plotkin y Eduardo Zimmermann (comps.), Los saberes de Estado, Buenos Aires, Edhasa, 2012: 35-62.

Terán, Oscar, Vida intelectual en el Buenos Aires de fin-de-siglo (1880-1910), Buenos Aires, Fondo de Cultura Económica, 2000.

Terán, Oscar, Positivismo y nación en la Argentina, Buenos Aires, Puntosur, 1987.

Zimmermann, Eduardo A., Los liberales reformistas. La cuestión social en la Argentina, 1890-1916, Buenos Aires, Editorial Sudamericana, 1995.

Fecha de recepción: 5/11/12

Fecha de aceptación: 30/1/13

\section{The World as an observation workshop. The creation of a National Department of Labor and international influences.}

This work analyzes the international experiences that influenced the formulation of the first responses that were tried out by the Argentinean State in the field of Labor legislation. The absence of a corpus of specialized knowledge (reliable statistics, reports, qualitative data) led the sectors concerned about resolving the workers' issue to study and observe the practices and methods developed in other countries. The experience acquired in that workshop of worldwide observation, within a context of a fluid international flow of ideas, models of analysis and theories, made it possible to devise and apply the first workers' laws as well as setting up the first labor organization in the country.

KeY WORDS: Social issues; Legislation; Flow of ideas; Knowledge; Codes; Labor policies. 